\title{
Editorial
}

\section{Open Sesame: a new generation journal}

\author{
Kamaldeep Bhui, Gin S. Malhi and Kenneth R. Kaufman
}

\section{Summary}

The Royal College of Psychiatrists welcomes you to its newest peer-reviewed research journal, British Journal of Psychiatry Open (BJPsych Open), which has been created to maximise the college's efforts to publish and disseminate the most exciting and progressive research in psychiatry and allied disciplines. BJPsych Open will maintain the same high quality standards established by the British Journal of Psychiatry (BJPsych). As an online journal without print restrictions, BJPsych Open will be able to publish many more excellent articles and article types not currently accommodated within BJPsych. The breadth of BJPsych Open is outlined in 'What we publish' and its strength is realised in our first issue, 'Powerful papers'. As editors, we welcome both your submissions and comments as our new generation journal grows.

\section{Declaration of interest}

None.

\section{Copyright and usage}

(c) The Royal College of Psychiatrists 2015. This is an open access article distributed under the terms of the Creative Commons Non-Commercial, No Derivatives (CC BY-NC-ND) licence.

\begin{abstract}
Kamaldeep Bhui (pictured) is Professor of Cultural Psychiatry \& Epidemiology at Queen Mary University of London, an Honorary Consultant Psychiatrist in the East London Foundation Trust and head of department at Barts Medical School. He is Editor of the British Journal of Psychiatry and BJPsych Open as well as the International Journal of Culture and Mental Health. Gin S. Malhi is Chair of Psychiatry at the University of Sydney, and Deputy Editor of BJPsych Open and Editor of the Australian and New Zealand Journal of Psychiatry. Kenneth R. Kaufman is Professor of Psychiatry, Neurology and Anesthesiology at Rutgers Robert Wood Johnson Medical School (New Brunswick) and Deputy Editor of BJPsych Open.
\end{abstract}

Welcome to the British Journal of Psychiatry Open, the new generation peer-reviewed research journal of the Royal College of Psychiatrists. BJPsych Open maintains the same high quality as the British Journal of Psychiatry, including rigorous academic oversight from the editorial board of the BJPsych along with a new dedicated team of highly skilled editors to maximise the scientific impact of this new online journal. There is more space to publish many more excellent articles than is usually possible in our print editions, not least because of the advantages of a digital journal. As a charity, the Royal College of Psychiatrists is committed to high scientific quality as well as public education and engagement and BJPsych Open will help us fulfil these commitments.

\section{Why publish in BJPsych Open?}

BJPsych Open will publish the most exciting and progressive research in all fields of psychiatry and allied disciplines. The journal will provide rapid peer review and decisions, leading to online publication within 28 days from acceptance. Therefore we will publish all year round, with online editions assembled quarterly. This provides greater efficiency for authors by maximising dissemination and sharing of research while maintaining the highest standards of scientific peer review and publication ethics. We anticipate publishing many different article types that are not currently accommodated within a print journal. Accepted manuscripts will be published under a Creative Commons license so permitting authors to continue to use their work freely without restriction.

\section{What we publish}

BJPsych Open offers publication of specialist research, and articles on clinical practice, ethics, research design, informed consent and innovations in research practice, including pilot and feasibility studies that offer significant advances in knowledge. Protocols of commissioned studies that have already undergone peer review are also eligible. Furthermore, BJPsych Open aims to accommodate high-quality submissions from low- and middle-income countries that continue to be underrepresented in the best research print journals. BJPsych Open is seeking papers from multiple disciplines and traditions of mental health research and practice, ranging from the environmental, social and cultural aspects through to nosology, epidemiology, neuroscience and biological research. Studies from the worlds of economics, medical humanities, geography and the arts as well as papers that offer critical and refreshing insights into the history and philosophies of care and clinical practice are welcome. Further, BJPsych Open will selectively consider unique and well-documented case reports addressing previously unknown efficacy, adverse events, drug interactions and novel insights into pathogenesis which have an impact on clinical practice and potential further research. The highest standards of accessible and provocative thinking, academic practice and innovations will be showcased.

\section{Powerful papers}

This first issue provides a great start. The role of informed consents and placebos in randomised controlled trials (RCTs) is a contested and underresearched area. ${ }^{1,2}$ We welcome critiques about the suitability and design of RCTs for testing interventions to improve mental health, and the alternative designs that perhaps provide more depth and information about mechanisms and contexts. The premature mortality of people with severe mental illness is now a well-established research finding, but the mechanisms, preventive interventions and personality factors require more investigation. For example, the paper by Prins et al $\mathrm{l}^{3}$ provides new evidence of significant psychopathology for patients with asthma that is not recognised or treated, including $20 \%$ of such patients having a personality disorder. Clearly there 
are implications not only for asthma management but also for the better management of psychopathology in medical practice. Finally, Trotta et al's study ${ }^{4}$ shows that parental psychosis as a measure of familial liability to psychosis and childhood adversity are each risk factors for future psychosis but do not combine or interact beyond their individual effects, suggesting different pathophysiological mechanisms.

We hope you enjoy this first issue and the benefits of publishing your best work in BJPsych Open.

Kamaldeep Bhui, Editor in Chief, Professor of Cultural Psychiatry and Epidemiology at Queen Mary University of London, Honorary Consultant Psychiatrist, East London Foundation Trust, London, UK; Gin S. Malhi, Deputy Editor, Chair of Psychiatry, University of Sydney, Australia; Kenneth R. Kaufman, Deputy Editor, Professor of Psychiatry, Neurology and Anesthesiology, Rutgers Robert Wood Johnson Medical School, New Brunswick, New Jersey, USA.

Correspondence: Kamaldeep Bhui, Barts and The London School of Medicine and Dentistry, Old Anatomy Building, Charterhouse Square, London EC1M 6BQ, UK. Email: k.s.bhui@qmul.ac.uk

First received 17 Jun 2015, accepted 17 Jun 2015

\section{References}

1 Chen SC, McCullumsmith C, Kim SYH. Disclosing the potential impact of placebo controls in antidepressant trials. BJPsych Open 2015; 1: 1-5. (doi:10.1192/bjpo.bp. 115.000109).

2 Kaufman KR. Ethical considerations in placebo-controlled randomised clinical trials (editorial). BJPsych Open 2015; 1: e3-e4. (doi:10.1192/bjpo.bp.115.000919).

3 Prins LCJ, van Son MJM, van Keimpema ARJ, Meijer J-WG, Buhring MEF, Pop VJM Unrecognised psychopathology in patients with difficult asthma: major mental and personality disorders. BJPsych Open 2015; 1: 14-17. (doi:10.1192/bjpo.bp.115. 000182).

4 Trotta A, Di Forti MM, lyegbe C, Green P, Dazzan P, Mondelli V, et al. Familial risk and childhood adversity interplay in the onset of psychosis. BJPsych Open 2015; 1: 6-13. (doi:10.1192/bjpo.bp.115.000158). 